\title{
Active Thermography for non-invasive panel paintings inspection: Absolute Thermal Contrast analysis of an artwork
}

\author{
by M. Rippa*, V. Pagliarulo*, A. Lanzillo**, G. Fatigati**, M. Grilli**, P. Ferraro* and P. Mormile* \\ *Institute of Applied Sciences and Intelligent Systems "E. Caianiello" of CNR, Via Campi Flegrei 34, \\ Pozzuoli (Na), Italy, m.rippa@isasi.cnr.it \\ ** University Suor Orsola Benincasa, Corso Vittorio Emanuele 293, Naples, Italy
}

\begin{abstract}
Active thermography is a well-known non-invasive imaging technique that represents a reliable means for providing a fast and low-cost analysis of an artwork. In this study, experimental data from a panel painting, representing an original artwork dating from the 16th and 17th centuries, was acquired in situ by pulsed thermography and processed by absolute thermal contrast analysis. Our results confirm as active thermography is a powerful technique to achieve valuable pre-restoration information concerning the detection and characterization of various types of defects present on the painting layer and a suitable tool for in situ analysis of artworks.
\end{abstract}

\section{Introduction}

In the last decades there has been a growing interest in the conservation of works of art belonging to cultural heritage and in the examination techniques preceding their restoration [1, 2]. In the case of painting on wood, different factors could affect their physical state like: fluctuations of ambient parameters as temperature, humidity and air pollutants, inappropriate light exposure or presence of bacteria. These factors could modify the artwork colours, cause expansions and contractions of different regions of the painting producing deformations, detachments and cracks of the panel or of the painting layer and more in general they increase its degradation. This deterioration process can become irreversible in time and, hence, it is important to detect and prevent their formation as early as possible using non destructive methods of investigation.

In this contest, the support of a professional scientific analysis, based on advanced devices techniques, is useful and, more and more often, relevant. For panel painting analysis, several diagnostic techniques are used but most of these are difficult to apply in situ mainly because of high costs and the strict stability conditions required. Active thermography (AT) is a well-known contact-less and non-invasive imaging technique, it is fast and of easy implementation and it represents a reliable means of providing a low-cost in situ analysis of an artwork [3-6]. In this technique, the surface of the object under investigation is stimulated using an external source in order to produce a dynamic thermal response that can be detected and recorded with an infrared camera. AT allows a rapid detection of a wide variety of defects such as voids, inclusions, delaminations, cracks, detachments, presence of nails and any type of anomaly that determines a change in thermo-physical properties of the painting under investigation. In this work, we used Pulsed Thermography (PT) to inspect in situ an artwork made on a wooden panel preserved at the University "Suor Orsola Benincasa" of Naples (Italy) and datable between the 16th and 17th centuries. PT is a fast and simple active thermography method in which the surface of the sample under investigation is heated using an energy pulse made with a lamp and the thermal response achieved is recorded during and after it. Parameters characterizing the thermal stimulation protocol were preliminary optimized in laboratory using panel paintings samples expressly made and reproducing the physical and structural characteristics of the original artwork. Absolute thermal contrast method was used to analyse the acquired data. Our measurements pointed out the presence of different type of defects and anomalies in the painting layer, allowing an assignment of their nature and hence providing important pre-restoration information.

\section{Materials and methods}

For PT technique an halogen lamp with tunable power was used as source to induce thermal pulses of the order of seconds on the investigated area. Illuminance incident on the samples was measured using a photo-radiometer (Delta Ohm HD 2102.2) with a lux meter probe (LP 471 PHOT). The thermal response achieved during and after heating was recorded with a frame rate of $10 \mathrm{~Hz}$ using a MWIR camera FLIR X6580 sc with a cooled indium antimonide (InSb) detector (spectral range 1.5-5.4 $\mu \mathrm{m}$, FPA $640 \times 512$ pixels and NETD $\sim 20 \mathrm{mk}$ at $25^{\circ} \mathrm{C}$ ) mounting a $50 \mathrm{~mm}$ focal lens with spectral band $3.5-5 \mu \mathrm{m}$ and IFOV $0.3 \mathrm{mrad}$. The artwork analysed in situ is an oil painting realized on a supporting structure in poplar wood of dimensions $89 \mathrm{~cm} \times 111.5 \mathrm{~cm} \times 3 \mathrm{~cm}$. It is a representation of the Eternal Father of an unknown author (figure 1-a). Its stylistic analysis leads to placing the work in the 16th-17th century. Preliminary to the analysis of the artwork, parameters concerning the thermal stimulation were optimized in laboratory using panel paintings samples (dimensions $20 \mathrm{~cm} \times 20 \mathrm{~cm} \times 3 \mathrm{~cm}$, figure 1-b) made with preparations techniques and pigments similar to 
those of the original artwork deduced from a precedent X-ray fluorescence (XRF) analysis. In figure 1-c are shown images of the experimental set-up used.

a

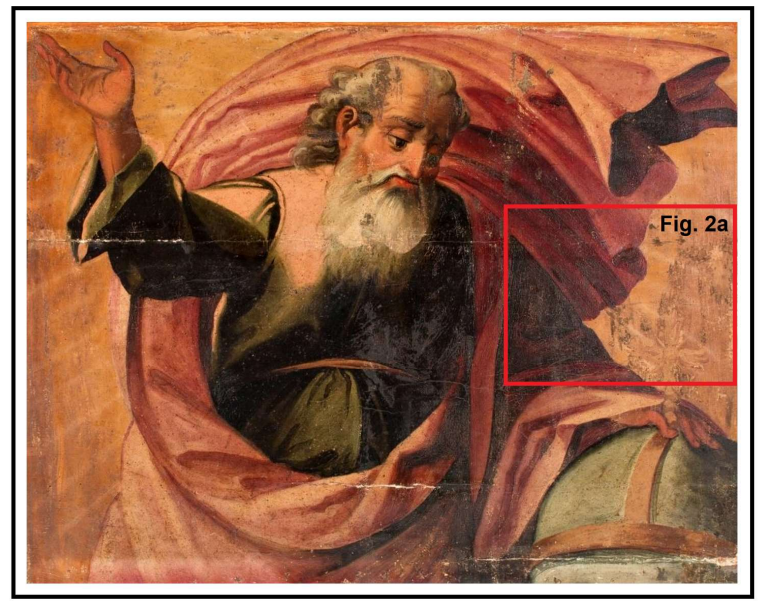

b

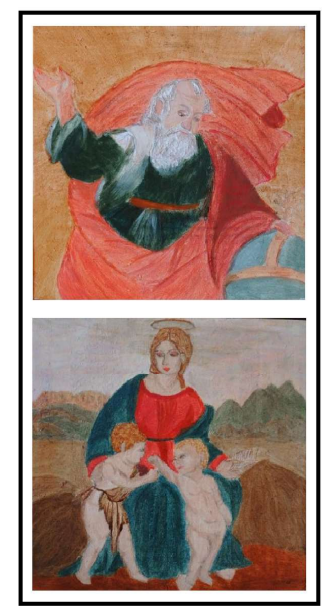

C

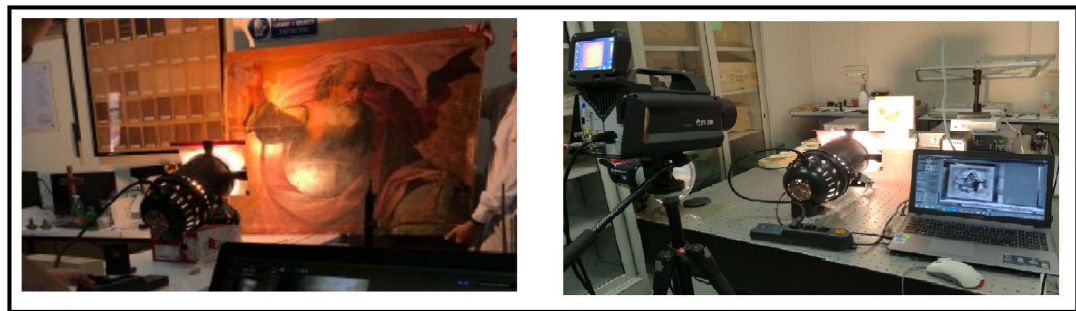

Fig 1: a) Artwork analysed represented by an oil painting on poplar wood, b) panel paintings samples, c) images of the experimental set-up used in situ (left) and in laboratory (right).

\section{Results and discussion}

The protocol of the thermal investigation to adopt for non-invasive analysis on the real artwork was studied and optimized, as a first step, on the painting samples expressly prepared. With optimized parameters, we mean a suitable setting that allows to get a heating as homogeneous as possible of the investigated area and a thermal excitation such to induce for the higher emissivity colours of the painting a maximum temperature increasing $\left(\Delta \mathrm{T}_{\max }\right)$ of $3^{\circ} \mathrm{C}$ (safety limit) and, at the same time, for the lower emissivity colours a minimum temperature increasing $\left(\Delta \mathrm{T}_{\min }\right)$ of $1^{\circ} \mathrm{C}$ necessary to perform their inspection. After several test performed changing the parameters values, we found as an optimal setting: a pulse time for the thermal stimulation of $10 \mathrm{~s}$, an illuminance of $400 \mathrm{~lx}$ and a distance between lamp and artwork of $65 \mathrm{~cm}$. Using these parameters, we performed the PT on the artwork in situ. In particular, we divided the artwork in 9 areas of investigation of dimension of about $30 \mathrm{~cm} \times 37 \mathrm{~cm}$ and we applied the stimulation protocol on each of it. We recorded the thermal behaviour of the different area during and after the thermal excitation. Successively, we analysed the movies registered frame by frame and calculated the absolute thermal contrast $\Delta T(t)=T_{D}(t)-T_{\text {sa }}(t)$, where $T_{D}(t)$ is the temperature trend of the defective area and $T_{s a}(t)$ that one of a sound area appropriately chosen. The inspection of Infrared images highlighted clearly the presence of different types of defects present in the painting layer, in particular: presence of small holes and nails, detachments, areas with thinner paint layers and some pentimenti. The various defects show a characteristic thermal response that can help in their identification and assignment. As an example in figure 2-a is shown the thermal image of one of the investigated areas and the $\Delta T(t)$ trend of three different defects visible in the image: A) a nail (red line), B) a detachment (blue line) and C) thinner paint layers (black line). The three trends are very different, in particular the higher thermal conductivity of the nail allows a faster trend of heating/cooling respect to that shown by detachment while the smaller amount of absorbent material (pigments) in the area with thinner paint layers produces a temperature increase lower than the sound area (negative $\Delta T(t)$ ). As another example of our results, in figure 2-b we report the visible and thermal images of the hand of the Eternal Father represented in the painting. Comparing the two images it is possible to notice a pentimento of the artist which originally painted the fingers with different positions and dimensions. 
a

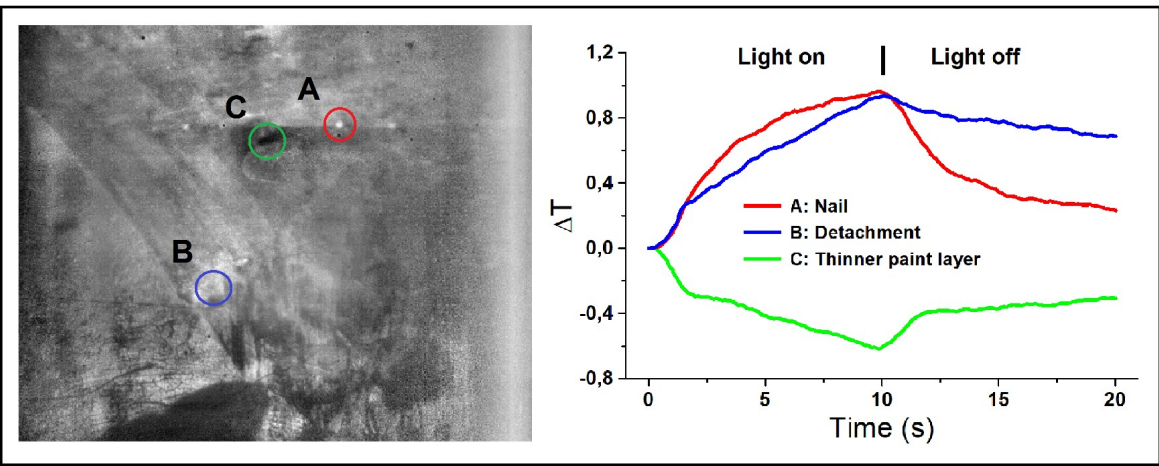

b

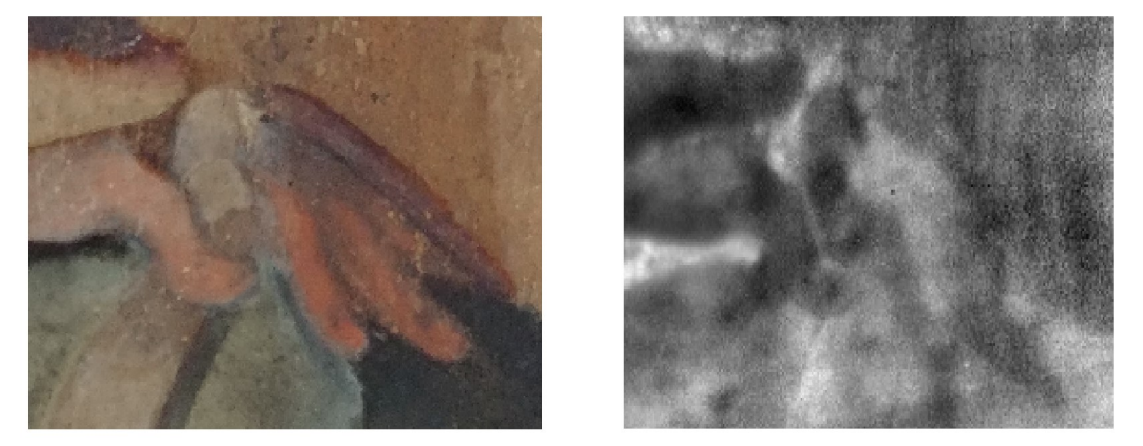

Fig 2: a) Thermogram of the area of the artwork indicated with a red rectangle in figure 1-a (left) and thermal contrast trend relative to three defects indicated in the thermogram with $A, B$ and $C$ (right), b) visible and thermal image of the hand of the subject.

\section{Conclusion}

Active thermography inspection is a powerful and efficient technique to achieve valuable information concerning the condition of paintings. Our results show that information on the presence of various types of defects can be obtained by thermographic measurements without performing complex analysis procedures and they confirm as this technique represents a suitable tool for non invasive panel paintings inspection in situ.

\section{REFERENCES}

[1] D. Gavrilov, R.Gr. Maev, D.P. Almond, "A review of imaging methods in analysis of works of art: Thermographic imaging method in art analysis". Can. J. Phys. Vol. 92, pp. 341-364, 2014.

[2] A. Bendada, S. Sfarra, C. Ibarra-Castanedo, M. Akhloufi, J.P. Caumes, C. Pradere, J.C. Batsale, X. Maldague, "Subsurface imaging for panel paintings inspection: A comparative study of the ultraviolet, the visible, the infrared and the terahertz spectra". Opto-Electron. Rev., 23, no. 1, 2015.

[3] S. Laureti, H. Malekmohammadi, M.K. Rizwan, P. Burrascano, S. Sfarra, M. Mostacci, M. Ricci, "Looking Through Paintings by Combining Hyper-Spectral Imaging and Pulse-Compression Thermography". Sensors, Vol. 19, no. 4335, pp 1-24, 2019.

[4] C. Ibarra-Castanedo, S. Sfarra, D. Ambrosini , D. Paoletti , A. Bendada, X. Maldague, "Infrared vision for the nondestructive assessment of panel paintings". CINDE Journal, Vol.31, no.5, 2010.

[5] C. Ibarra-Castanedo, S. Sfarra, D. Ambrosini , D. Paoletti, A. Bendada, X. Maldague, "Subsurface defect characterization in artworks by quantitative pulsed phase thermography and holographic interferometry". Quantitative InfraRed Thermography Journal. - Vol. 7, no 1, pp. 85-114, 2010.

[6] R. Usamentiaga, P. Venegas, J. Guerediaga, L. Vega, J. Molleda, F.G. Bulnes, "Infrared Thermography for Temperature measurements and Non-DestructiveTesting". Sensors, 14, 12305-12348, 2014. 